\title{
The cannabinoids - important therapeutic approach in the field of oncology
}

\author{
Nicoleta Mirela BLEBEA ${ }^{1}$, Laura Adriana BUCUR², Simona NEGRES ${ }^{3}$ \\ 1 "Carol Davila" University of Medicine and Pharmacy, Bucharest, Romania \\ ${ }^{2}$ Disciplines of Pharmacognosy and Clinical Pharmacy, Faculty of Pharmacy, "Ovidius" University, Constanta,
}

Romania

${ }^{3}$ Department Discipline of Pharmacology and Clinical Pharmacy, Faculty of Pharmacy, "Carol Davila" University of Medicine and Pharmacy, Bucharest, Romania

\begin{abstract}
Oncological diseases are the most common cause of death worldwide, it is estimated that $25 \%$ of the population will face the diagnosis of cancer during life. Phytochemicals such as cannabinoids (CBs) have been used in various branches of medicine for their properties, and the discovery of the anti-tumor, anti-emetic and anti-inflammatory effects of some of these substances has encouraged their use in oncology. Phytocannabinoids, cannabidiol (CBD) and $\triangle$-9-tetrahydrocannabinol (THC), have numerous anti-emetic, analgesic, orexigenic, anti-inflammatory / immunosuppressive pharmacodynamic effects. In recent years, studies have been aimed at evaluating their efficacy as antineoplastic agents. Much in vivo and in vitro research has demonstrated the efficacy of CBs on certain tumor cell lines, highlighting their potential role in the complementary treatment of cancer. This paper suggests that exploring the molecular mechanisms induced by CBs in cancer cells may contribute to the development of effective treatments in oncological diseases.
\end{abstract}

Keywords: CBD, THC, phytochemicals, cannabinoids (CBs), oncology

\section{INTRODUCTION}

The cannabis plant has been used for ages in medical therapy. People have been using cannabis for over 5,000 years, the first the discoveries are from China. It has been used to treat gout, malaria, constipation, rheumatism and to produce fiber, and seeds [1]. Cannabis extracts contain a large number of phytochemicals such as terpenes, flavonoids, and CBs. Cannabis derivatives include the bioactive constituents of cannabis (THC, CBD), endogenous lipids with cannabinoid-like activity, anandamide (AEA), 2-arachydonoyl-glycerol (2-AG) and the synthetic analogues WIN55,212-2, 2-methyl-2'-Fanandamide (Met-F-AEA), JWH-015, HU-210, HU-331
[2]. However, cannabis contains more than 60 active principles. CBs have enjoyed great interest in recent years due to various pharmacological actions. There are over than a hundred components different CBs, the main ones being THC with a psychotropic effect, and CBD with non-psychotropic effects. Unlike THC, CBD does not produce euphoria, but has been shown to modulate THC activity and has anti-inflammatory and analgesic activities [3-5]. THC, in the synthetic form known as dronabinol (Marinol ${ }^{\circledR}$ ), has been approved by the Food and Drug Administration (FDA) for antiemetic and stimulation of appetite, used by patients with AIDS and patients with loss of body weigh [6-9]. Currently, nabilone (Cesamet ${ }^{\circledR}$ ), a 
synthetic analogue of THC, is used to suppress nausea and vomiting caused by chemotherapy. Recently, CBD has been approved for the treatment of pediatric seizures in the United States (U.S.) [10]. CBD is used as active ingredient in the following products, Epidiolex ${ }^{\circledR}$ - oral solution (contains only CBD) and Sativex ${ }^{\circledR}$ - oralmucosal spray (contains both CBD and THC) for improving spasticity in patients with multiple sclerosis. CBD products could be used off-label for the treatment of other medical conditions [11-15]. At the same time, studies using CBs for the treatment of oncological diseases have given promising results in terms of antitumor activity [16-19]. Moreover, in addition to their inhibitory effects on tumor growth and migration, angiogenesis and metastasis, the ability of these compounds to induce various cell death pathways has been highlighted. Research suggest that cannabinoid receptor agonists may offer a new strategy for treating cancer [20].

Cancer is one of the leading causes of death worldwide [21]. Phytocannabinoids, CBD and THC reduce nausea and vomiting, especially during chemotherapy. This is due to their ability to reduce the release of serotonin from enterochromafin cells in the small intestine [22]. Because CBD does not induce psychotropic effects, unlike other $\mathrm{CBs}$, its use as an anti-emetic is of great interest [23]. CBs, such as CBD and $\mathrm{THC}$, have demonstrated antitumor properties in vivo and in vitro in melanoma [24]. THC induces apoptosis, and its effects are increased in concomitant treatment with $C B D$, which may increase the chemotherapeutic effect of cisplatin in animal models with melanoma [25]. In addition, synergies have been demonstrated between CB and antineoplastic agents (temozolomide, paclitaxel) [26]. This paper suggests that exploring the molecular mechanisms induced by CBs in cancer cells may contribute to the development of effective treatments in oncological diseases.

\section{ROLE OF CANNABINOIDS IN CANCER}

The endocannabinoid system (ECS) consists of CB1 and $\mathrm{CB} 2$ receptors, endocannabinoids (eCBs), endogenous ligands the associated enzymatic machinery (transporters, biosynthetic and degradative enzymes). Our body naturally produces cannabinoidlike compounds known as eCBs. These substances have specific receptors called $C B 1$ and $C B 2$ receptors that are found throughout the body and in the brain. The cannabinoid receptors, CB1 and CB2 are $\mathrm{G}$ protein-coupled receptors. The CB1 receptor is located on central and peripheral neurons, while the $\mathrm{CB} 2$ receptor is located on immune cells. Much of the calming, anti-inflammatory and psychoactive effects of $\mathrm{CBs}$ are mediated by $\mathrm{CB} 1$ and $\mathrm{CB} 2$ receptors [25]. Phytocannabinoids and synthetic CBs interact with the components of ECS, thus affecting the progression of oncological diseases. In cancer patients, CBs has been used primarily as part of palliative care. In vitro and in vivo cancer models show that CBs can modulate tumor growth, however, the antitumor effects are dependent on the type of cancer and the concentration of the drug. Understanding how CBs are able to regulate cellular processes involved in tumorigenesis, such as cell cycle progression, cell proliferation and cell death, and the interactions between CBs and the immune system, are essential for cancer patients [25]. In 1975, Munson et al. demonstrated the antineoplastic activity of $C B$ by inhibiting the growth of Lewis lung carcinoma after oral administration to mice. The potential antitumor activity of phytocannabinoids, (-) - trans- $\Delta 9$ tetrahydrocannabinol (trans- $\triangle 9-\mathrm{THC}$ ), cannabinol (CBN), $\triangle 8$-tetrahydrocannabinol ( $\triangle 8$-THC), CBD and cannabicyclol ( $\mathrm{CBL})$, as well as synthetic $\mathrm{CBs}$ are at the center of current research $[27,28]$.

Research has shown that CBs exert anti-proliferative and proapoptotic effects in different types of cancer (breast, lung, glioma, thyroid, lymphoma, skin, pancreas, uterus, prostate, bladder and colorectal carcinoma) both in vitro and in vivo $[27,29]$. It seems that the balance between the protumorigenic and antitumor effects of CBs depends, as previous studies have shown, on their concentration [25].

For example, Hart et al. showed that treatment of glioblastoma and lung carcinoma with nanomolar concentrations of THC (instead of commonly used micromolar concentrations) leads to increased cell proliferation. Therefore, in the therapy of oncological diseases, it is very important to consider the risk of accelerating tumor growth due to the proliferative potential of CBs concentration $[27,30]$.

The most common cancer in men is prostate cancer, being one of the leading causes of death in men of all races. $C B 1$ and $C B 2$ levels were elevated in prostate cancer and in several cell lines compared to normal prostate epithelial cells. Sharma et al. showed in a study on human prostate cancer cell lines, that CBD is a potent inhibitor of cancer cell growth, while this 
potency was lower in non-cancer cells $[30,31]$. Additionally, treatment with CBD has been shown to decrease the expression prostate-specific antigen (PSA), vascular endothelial growth factor (VEGF), and pro-inflammatory cytokines.

In their study Ravi et al. investigated the combination of Met-F-AEA and selective irreversible fatty acid amid hydrolase inhibitor (FAAH), showed that they synergistically inhibited the proliferative and chemotactic activity (EGF) induced by the epidermal growth factor of lung cancer [32].

In the U.S. colorectal cancer (CRC) is the third leading cause of cancer deaths in both men and females. Several studies have also investigated CBD as an adjunct to chemotherapy for CRC [33]. Cannabigerol (CBG) is a safe non-psychotropic CBs, which interacts with specific targets involved in carcinogenesis. CBG inhibits the growth of CRC cells mainly through a pro-apoptotic mechanism and prevents the development and growth of the colon carcinogenesis in vivo [17]. In vitro studies support beneficial effect of CBD in CRC cell lines: CBD protects DNA from increased oxidative damage low concentrations of eCBs and tumor cells proliferation. CBD has been reported to have various biological activities, including an antiproliferative effect on cancer cells, although CBD is obtained from non-enzymatic decarboxylation of cannabidiolic acid (CBDA). The study presented by Takeda et al. suggests for the first time that CBDA inhibits the migration of breast cancer cells, apparently through a mechanism involving the inhibition of protein kinase A (PKA) coupled with the activation of the small GTPase [34].

Glioma is the most common primary brain malignancy. CBD has been shown to successfully inhibit cell death in vitro cell migration and invasion, decrease tumor size, vascularity, growth and weight, and increases survival and induces tumor regression in vivo [33].

Many studies have investigated the ability of phytocannabinoids, $\mathrm{CBD}$, or THC to reduce the viability of cancer cells in breast cancer, colorectal cancer, glioblastomas, and lung cancer $[17,35,36]$. Breast cancer is the number one leading cause of new cancer cases and the second leading cause of cancer deaths of women in the U.S. Taken with cisplatin, CBD has been shown to be effective on many breast cancer cells, it appears to be the next step in the development of CBD as an alternative treatment for breast cancers [33].
The antitumor effect of CBs in bladder cancer is very little known. Tomko et al. demonstrated that CBD, THC and other $\mathrm{CBs}$ reduce the cell viability of cancerous lines in the bladder. Their combination with gemcitabine or cisplatin may also induce different responses, depending on concentrations used. CBD and THC have synergistic properties with other CBs: cannabichromene and cannabivarin [37].

One of the diseases with the highest mortality rate is lung cancer. CBs could stop tumor growth in this condition without side effects, specifically targeting CB1 / CB2 receptors. Chakravarti et al. in their studies, suggest the involvement of cyclooxygenase-2 (COX-2) and peroxisome proliferator-activated receptors (PPAR- $\gamma$ ) in the proapoptotic and tumor regressive action of CBD $[32,38]$.

Oral cancer occurs when the disease spreads to the mouth, tongue, lips and throat. THC induces apoptosis in oral squamous cell carcinoma [39].

Dando et al. studied the antitumor effects of CBs and described them in various oncological disease, including pancreatic adenocarcinoma. To demonstrate potential pathways that mediate cannabinoiddependent inhibition of pancreatic cancer cell growth, they used CBs specific for $\mathrm{CB} 1$ and $\mathrm{CB} 2$ receptors and metabolomics assays. Thus, it has been shown to induce autophagy and inhibit cell growth by CBs [40].

Regarding thyroid cancers, CBD caused an antiproliferative by activation of apoptosis and cell cycle arrest. This effect was observed due to the activation of cannabinoid receptors. Chakravarti et al. in their studies, they reported that CB2 agonist JWH-133 and CB1 / CB2 agonist WIN-55,212-2 induced apoptosis in $A R O$ and ARO / IL-12 cells. The analogue of anandamide, Met-F-AEA, also induced apoptosis in thyroid carcinoma cells $[33,38]$.

\section{EFFECTS ON INVASION AND METASTASIS}

CBs affect markers associated with cancer invasion and metastasis, including markers of migration, adhesion, invasion, and metastasis. Migration studies have shown that AEA, 2-AG, CBD, HU-210, JWH-133, Met-F-AEA, THC and WIN-55,212-2 all reduced migration or markers. migration across a wide range of cell lines [41]. Bone metastases are a common complication of oncological diseases. Breast cancer 
and prostate cancer metastasize mainly to the bones, acting as a fertile ground for the development of secondary tumors. Patients with bone metastases have severe pain, so CBs can be used to relieve pain. Migration and cell invasion from breast cancer to other organs (e.g. lungs) are inhibited by Met-F-AEA, WIN 55,212-2, JWH-133 and JWH-015 [38]. CBD inhibits cell proliferation and the metastatic cell line of the breast and reduces the volume of the primary tumor. Moreover, CBD reduced the number of metastatic outbreaks. According to the studies of Chakravarti et al. JWH-015 and Win55, 212-2 inhibit in vitro chemotaxis, and tumor growth in vivo and lung metastases by inhibiting AKT, but selective pretreatment of CB1 / CB2, AM251 and AM630 antagonists antagonized their effects. THC inhibits the growth of pulmonary adenocarcinoma by inhibiting DNA synthesis and suppresses the growth and metastasis of A549 and SW-1573 in vivo and in vitro [38]. All of these results suggest that CBs have the potential to reduce the development, growth and metastasis of cancer.

\section{CONCLUSIONS}

CBs have been used for their pharmaceutical properties since ancient times. The anti-emetic, anti-inflammatory and anti-carcinogenic properties of various CBs make them good candidates for the complementary treatment of various, oncological diseases.

In conclusion, $\mathrm{CBs}$, especially synthetic ones, have antitumor effects such as inhibition of cell growth, viability, proliferation and invasion, increased apoptosis and suppression of specific proinflammatory cytokines. These are similar to the antitumor effects of plant CBs. Moreover, synthetic CBs have the potential to be more selective and stronger than their natural counterparts, which is why they represent an important therapeutic approach in the field of oncology.

Conflict of interest: none declared Financial support: none declared

\section{REFERENCES}

1. Ambrose T, Simmons A. Cannabis, cannabinoids, and the endocannabinoid system-is there therapeutic potential for inflammatory bowel disease? J Crohns Colitis. 2019;13(4):525535.

2. Calvaruso G, Pellerito O, Notaro A, et al. Cannabinoid-associated cell death mechanisms in tumor models (review). Int J Oncol. 2012;41(2):407-413.

3. Burstein S. Cannabidiol (CBD) and its analogs:a review of their effects on inflammation. Bioorg Med Chem. 2015;23(7):1377-1385

4. Hammell DC, Zhang LP, Ma F, et al. Transdermal cannabidiol reduces inflammation and pain-related behaviours in a rat model of arthritis. Eur J Pain. 2016;20(6):936-948.

5. 5. Philpott HT, O'Brien M, McDougall JJ. Attenuation of early phase inflammation by cannabidiol prevents pain and nerve damage in rat osteoarthritis. Pain. 2017;158(12):2442-2451.

6. Pharmaceuticals U. Marinol. New drug application. Food and Drug Administration:FDA;2004.

7. Pharmaceuticals V. Cesamet. New drug application. Food and Drug Administration:FDA;2006.

8. Navari R. Management of chemotherapy-induced nausea and vomiting:focus on newer agents and new uses for older agents. Drugs. 2013;73(3):249-262.

9. Argilés JM, Busquets S, Stemmler B, et al. Cancer cachexia:understanding the molecular basis. Nat Rev Cancer. 2014;14(11):754-762.

10. Pharmaceuticals G. Epidiolex. New drug application. Food and Drug Administration:FDA;2018.

11. Eržen M, Košir IJ, Ocvirk M, et al. Metabolomic Analysis of Cannabinoid and Essential Oil Profiles in Different Hemp (Cannabis sativa L.) Phenotypes. Plants. 2021.

12. Hanuš LO, Meyer SM, Muñoz E, et al. Phytocannabinoids:A unified critical inventory. Nat Prod Rep. 2016;33, 1357-1392.
13. Hazekamp A, Choi YH, Verpoorte R. Quantitative analysis of cannabinoids from Cannabis sativa using $1 \mathrm{H}-\mathrm{NMR}$. Chem Pharm Bull. 2004;52(6):718-721.

14. Sandler LN, Beckerman JL, Whitford F et al. Cannabis as conundrum. Crop Prot. 2019;117:37-44.

15. Blebea NM, Costache T, Negreș $S$, The qualitative and quantitative analysis of CBD in hemp oils by UHPLC with PDA and applications. Scientific Papers: Series D, Animal Science, 2019;62(1):138-142.

16. Greenhough A, Patsos HA, Williams AC, et al. The cannabinoid delta(9)-tetrahydrocannabinol inhibits RAS-MAPK and PI3K-AKT survival signalling and induces BAD-mediated apoptosis in colorectal cancer cells. Int J Cancer. 2007 121(10):2172- 2180.

17. Borrelli $F$, Pagano $E$, Romano $B$, et al. Colon carcinogenesis is inhibited by the TRPM8 antagonist cannabigerol, a cannabisderived non-psychotropic cannabinoid. Carcinogenesis. 2014;35(12):2787-2797.

18. Pellerito O, Notaro A, Sabella S, et al. WIN induces apoptotic cell death in human colon cancer cells through a block of autophagic flux dependent on PPARy down-regulation. Apoptosis. 2014;19(6):1029-1042.

19. Raup-Konsavage WM, Johnson M, Legare CA, et al. Synthetic cannabinoid activity against colorectal cancer cells. Cannabis Cannabinoid Res. 2018;3(1):272-281.

20. Pisanti S, Malfitano AM, Grimaldi C, et al. Use of cannabinoid receptor agonists in cancer therapy as palliative and curative agents. Best Pract Res Clin Endocrinol Metab. 2009;23(1):117-131.

21. Blebea NM, Bucur LA. Pharmacotherapeutic options in neoplastic diseases - part I. Farmacist.ro. 2021;198(1):18-20.

22. Mortimer TL, Mabin T, Engelbrecht AM. Cannabinoids:the lows and the highs of chemotherapy-induced nausea and vomiting. Future Oncol. 2019;15(9):1035-1049. 
23. Scheau C, Badarau IA, Mihai LG, et al. Emmerging applications of some important natural compounds in the field of oncology. Farmacia. 2020;68(6):984-991.

24. Scheau C, Mihai LG, Badarau IA, et al. Cannabinoids in the Pathophysiology of Skin Inflammation. Molecules, 2020;25(3):1-23.

25. Pertwee RG. The diverse $C B 1$ and $C B 2$ receptor pharmacology of three plant cannabinoids:delta9-tetrahydrocannabinol, cannabidiol and delta9-tetrahydrocannabivarin. Br J Pharmacol. 2008;153(2):199-215.

26. Miyato H, Kitayama J, Yamashita H, et al.Pharmacological synergism between cannabinoids and paclitaxel in gastric cancer cell lines. J Surg Res. 2009;155(1):40-47.

27. Dariš $\mathrm{B}$, Tancer Verboten $\mathrm{M}$, Knez Ž, et al. Cannabinoids in cancer treatment:Therapeutic potential and legislation. Bosn J Basic Med Sci. 2019;19(1):14-23.

28. Birdsall SM, Birdsall TC, Tims LA. The Use of Medical Marijuana in Cancer. Curr Oncol Rep. 2016;18(7):40.

29. Cridge BJ, Rosengren RJ, Critical Evaluation of the Potential Use of Cannabinoids in Cancer Management. Cancer Manag Res. 2013;5:301-313.

30. Hart S, Fischer OM, Ullrich A. Cannabinoids induce cancer cell proliferation via tumor necrosis factor alpha-converting enzyme (TACE/ADAM17)-mediated transactivation of the epidermal growth factor receptor. Cancer Res. 2004;64(6):1943-1950.

31. Sharma C, Sadek B, Goyal SN, et al. Small Molecules from Nature Targeting G-Protein Coupled Cannabinoid Receptors:Potential Leads for Drug Discovery and Development. Evid Based Complement Alternat Med. 2015;2015:238482.

32. Ravi J, Sneh A, Shilo K, et al. FAAH inhibition enhances anandamidemediated anti-tumorigenic effects in small cell lung cancer by down-regulating the EGF / EGFR pathway. Oncotarget. 2014;5(9):2475-2486.

33. Seltzer ES, Watters AK, MacKenzie D Jr, et al. Cannabidiol (CBD) as a Promising Anti-Cancer Drug. Cancers. 2020;12(11):3203.

34. Takeda S, Okajima S, Miyoshi H, et al. Cannabidiolic acid, a major cannabinoid in fiber-type cannabis, is an inhibitor of MDA-MB-231 breast cancer cell migration. Toxicol Lett. 2012;214(3):314-319.

35. Fonseca BM, Correia-da-Silva G, Teixeira NA. Cannabinoid-induced cell death in endometrial cancer cells:involvement of TRPV1 receptors in apoptosis. J Physiol Biochem. 2018;74(2):261-272.

36. Jeong S, Yun HK, Jeong YA, et al. Cannabidiol-induced apoptosis is mediated by activation of Noxa in human colorectal cancer cells. Cancer Lett. 2019;447:12-23.

37. Tomko A, Whynot E, Dupré D. (2021). Anti-cancer properties of cannabidiol and $\Delta 9$-tetrahydrocannabinol and potential synergistic effects with gemcitabine, cisplatin and other cannabinoids in bladder cancer. Biorxiv.2021;3.

38. Chakravarti B, Ravi J, Ganju RK. Cannabinoids as therapeutic agents in cancer:current status and future implications. Oncotarget. 2014;5(15):5852-5872

39. Lopes CF, de Angelis BB, Prudente HM, et al. Concomitant consumption of marijuana, alcohol and tobacco in oral squamous cell carcinoma development and progression:recent advances and challenges. Arch Oral Biol. 2012;57(8):1026-1033.

40. Dando I, Donadelli M, Costanzo C, et al. Cannabinoids inhibit energetic metabolism and induce AMPK-dependent autophagy in pancreatic cancer cells. Cell Death Dis. 2013;4(6):e664.

41. Cridge BJ, Rosengren RJ. Critical appraisal of the potential use of cannabinoids in cancer management. Cancer Manag Res. 2013;5:301-313. 